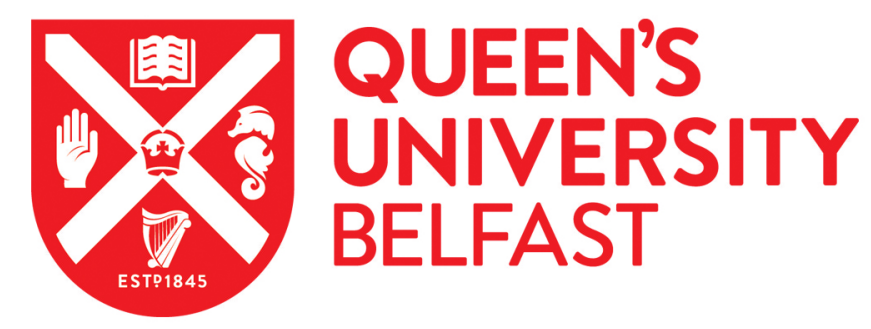

\title{
Characterisation of Passive Intermodulation in Passive RF Devices with X-parameter
}

Kozlov, D. S., Shitvov, A. P., \& Schuchinsky, A. G. (2014). Characterisation of Passive Intermodulation in Passive RF Devices with X-parameter. In 2014 Loughborough Antennas and Propagation Conference (LAPC) (pp. 64-67). Institute of Electrical and Electronics Engineers Inc.. https://doi.org/10.1109/LAPC.2014.6996321

Published in:

2014 Loughborough Antennas and Propagation Conference (LAPC)

Document Version:

Early version, also known as pre-print

Queen's University Belfast - Research Portal:

Link to publication record in Queen's University Belfast Research Portal

Publisher rights

( $) 2014$ IEEE. Personal use of this material is permitted. Permission from IEEE must be obtained for all other uses, in any current or future media, including reprinting/republishing this material for advertising or promotional purposes, creating new collective works, for resale or redistribution to servers or lists, or reuse of any copyrighted component of this work in other works

\section{General rights}

Copyright for the publications made accessible via the Queen's University Belfast Research Portal is retained by the author(s) and / or other copyright owners and it is a condition of accessing these publications that users recognise and abide by the legal requirements associated with these rights.

Take down policy

The Research Portal is Queen's institutional repository that provides access to Queen's research output. Every effort has been made to ensure that content in the Research Portal does not infringe any person's rights, or applicable UK laws. If you discover content in the Research Portal that you believe breaches copyright or violates any law, please contact openaccess@qub.ac.uk. 


\title{
Characterisation of Passive Intermodulation in Passive RF Devices with X-parameters
}

\author{
Dmitry S. Kozlov, Alexey P. Shitvov and Alexander G. Schuchinsky \\ Institute of Electronics, Communications and Information Technology (ECIT) \\ Queen's University Belfast \\ Queen's Road, Queen's Island, Belfast BT3 9DT, UK \\ d.kozlov@qub.ac.uk, a.shitvov@qub.ac.uk, a.schuchinsky@qub.ac.uk
}

\begin{abstract}
A novel approach to the modelling of passive intermodulation (PIM) generation in passive components with distributed weak nonlinearities is outlined. Based upon the formalism of $\mathrm{X}$-parameters, it provides a unified framework for co-design of antenna beamforming networks, filters, combiners, phase shifters and other passive and active devices containing nonlinearities at RF front-end. The effects of discontinuities and complex circuit layouts can be efficiently evaluated with the aid of the equivalent networks of the canonical nonlinear elements. The main concepts are illustrated by examples of numerical simulations of PIM generation in the transmission lines and comparison with the measurement results.
\end{abstract}

Keywords-intermodulation distortion; passive intermodulation (PIM); distributed nonlinearity; X-parameters

\section{INTRODUCTION}

Intermodulation is the phenomenon of generating spectral components with frequencies which are not present in the original signals [1]. Passive intermodulation (PIM) distortion caused by weak nonlinearities may severely deteriorate the receiver sensitivity, ultimately resulting in dramatic reduction of data throughputs in wireless communications systems. PIM products generated by passive components and circuits of the transmitter RF front-end increase interference and desensitize collocated base station receivers.

The requirements of signal integrity in the base stations for mobile and space communications impose increasingly stringent specifications of the PIM performance of passive devices such as multiband antenna arrays, beamforming networks, phase shifters and filters. Majority of these devices are still designed without considering their nonlinear response, and their PIM performance is assessed only experimentally using a basic two-tone CW test. However, such a test does not allow for location of PIM sources, nor an adequate nonlinear device characterisation in the case of complex waveforms. Therefore, it becomes of critical importance to incorporate the PIM distortion analysis into the RF front-end design process.

Various mechanisms of PIM in passive RF components, such as connectors, coaxial cables, filters, duplexers and printed circuit boards, have been investigated in the past. The main causes of passive nonlinearities are usually attributed to contact effects, soldered joints, electro-thermal phenomena, ferroic materials and protective coating.
The most commonly used passive components of the RF front-end, such as power combiners/dividers, phase shifters and filters, are composed of the transmission line (TL) sections and discontinuities such as line width steps, bends, junctions etc. Nonlinear characterisation of individual circuit elements and their assemblies is a prerequisite for predictive modelling of the PIM performance of the whole system. Addressing this challenging task will provide a deeper insight in the mechanisms of PIM generation in antenna systems and help devising a means for PIM mitigation.

Passive RF devices are usually characterised with the aid of the S-parameters. However, the conventional S-parameters are suitable only for linear circuits and cannot be directly applied to description of nonlinear components. To overcome these limitations, the concept of X-parameters was introduced as an extension of S-parameters for the analysis of harmonic generation and intermodulation distortion in active nonlinear devices [2, 3]. In fact, X-parameters represent a superset of the S-parameters, describing the nonlinear device response in both large- and small-signal regimes. They include magnitude and phase of the spectral components of higher harmonics and intermodulation products, in addition to the spectral content of the stimuli. To date, X-parameters have been mainly used for characterisation of harmonic generation by lumped nonlinear devices and stepped nonlinear TL (NTL) [4]. In this paper, the $\mathrm{X}$-parameter formalism is extended to the analysis of distributed PIM generation in passive circuits. The main concepts are outlined below and validity of the proposed approach is illustrated by examples.

\section{NONLINEAR MODEL OF DISTRIBUTED PIM GENERATION}

The formalism of X-parameters developed for the "black box" characterisation of nonlinear devices is primarily employed in retrieving the behavioural models of amplifiers and lumped nonlinearities. In order to apply the X-parameters to the analysis of PIM generation in passive components with distributed nonlinearities, an actual physical structure can be partitioned and represented as an ensemble of basic constituent elements. Once the electrical size of such individual elements is small as compared with a wavelength, they can be described by their equivalent lumped element circuits. For example, electrically short linear $R L C$ networks are often used for modelling TL, canonical discontinuities and their assemblies. Such approach can be extended to the PIM analysis of complex networks using X-parameters, retrieved 


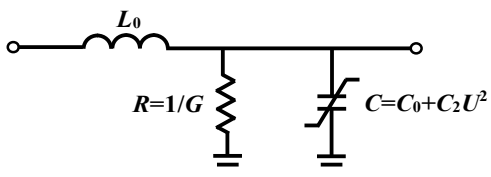

Fig. 1. An equivalent circuit of a unit cell of NTL

from the experimental measurements or simulations of canonical lumped and/or distributed nonlinearities.

It is necessary to remark, however, that the topology and size of the circuit constituent elements may considerably affect numerical accuracy and stability of such nonlinear network models. The issues have been discussed in [5] in the context of NTL discretisation. Here we consider a simple model of NTL, represented as a chain of unit cells each of which is represented by equivalent circuit shown in Fig. 1.

A length of uniform microstrip NTL has been analysed using the Harmonic Balance solver of Agilent ADS simulator. The NTL has been partitioned in short segments, each described by its equivalent $R L C$ circuit shown in Fig. 1. Nonlinear capacitor in this schematic represents a weakly nonlinear substrate which is described by the third order polynomial dependence on voltage across the capacitor. Such a model of nonlinearity is not restrictive, and is used here only to illustrate the main concepts.

The forward and reverse third order PIM (PIM3) products in NTL have been simulated with two $44 \mathrm{dBm}$ CW tones of frequencies $f_{1}=935 \mathrm{MHz}$ and $f_{2}=960 \mathrm{MHz}$. Electrical length of the primitive cells shown in Fig. 1 was initially chosen at $2^{\circ}$ at PIM3 frequency $2 f_{1}-f_{2}=910 \mathrm{MHz}$ that ensured better than

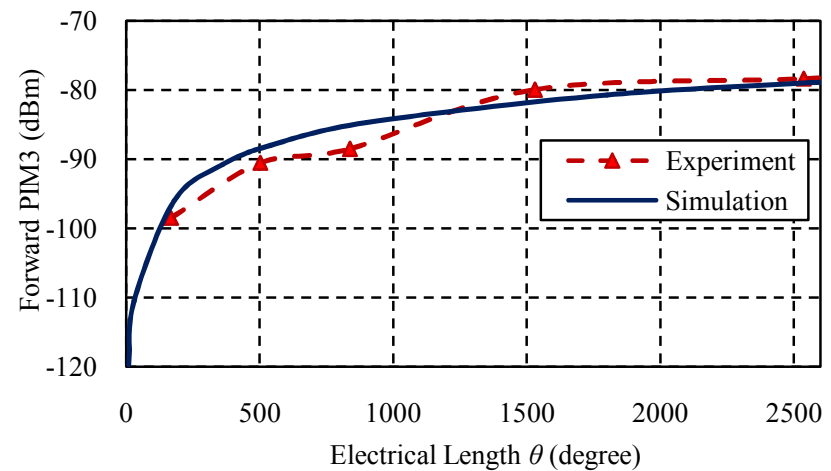

a)

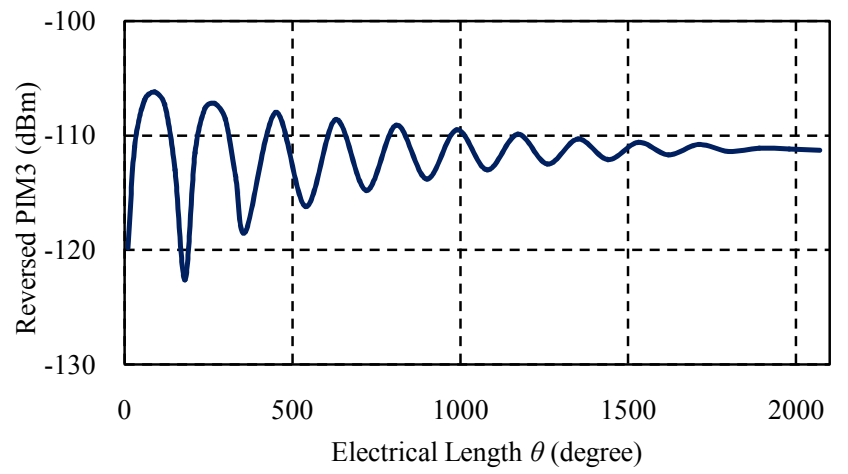

b)

Fig. 2. Forward (a) and reversed (b) PIM3 products versus electrical length of the nonlinear transmission line.
$30 \mathrm{~dB}$ return loss in the frequency band from $\mathrm{DC}$ to $3 \mathrm{GHz}$. Thishas been realised with the following circuit parameters: $L_{0}=0.3 \mathrm{nH}, \quad G=3 * 10^{-5} \mathrm{~S}, \quad C_{0}=0.127 \mathrm{pF}$. The nonlinear capacitance $C_{2}=2.11 * 10^{-11} \mathrm{pF} / \mathrm{V}^{2}$ has been retrieved from the experimental data reported in $[6,7]$.

The equivalent circuit model of uniform NTL has been validated by simulations of the characteristic features of distributed PIM3 generation, such as a cumulative growth of the PIM3 level at the NTL output ("forward PIM") and periodic undulations of the PIM3 level at the input ("reversed PIM") with the line length. This behaviour of PIM3 products is a manifestation of the phase synchronism in degenerative four-wave mixing process in NTL. The calculated PIM3 magnitudes are shown in Fig. 2 in dependence on the NTL electrical length $\theta$. Comparison of the simulated and measured forward PIM3 products in Fig. 2(a) demonstrates their very good correlation - both curves exhibit similar rates of the cumulative growth of the forward PIM3 products with the line length. The decaying periodic undulations of the reverse PIM3 level in Fig. 2(b) are also in full agreement with the qualitative analytical model of distributed PIM generation [6].

In order to assess accuracy of the NTL approximation by a cascade of the unit cells represented by the equivalent circuits of Fig. 1, simulations have been carried out with unit cells of several different electrical lengths. It has been found that coarser NTL partitioning decreases return loss from $43 \mathrm{~dB}$ to $24 \mathrm{~dB}$ when the unit cell length increases from $1^{\circ}$ to $10^{\circ}$. Fig. 3 shows PIM3 versus NTL length, simulated at different electrical lengths of the unit cells. It is evident that the unit cell length has negligible effect on the forward PIM3 products. However, reverse PIM3 is considerably more sensitive to the unit cell size. Coarser discretisation of NTL causes higher simulation error of reverse PIM3 generated in the NTL longer than half a wavelength. It is necessary to emphasise that PIM3 dependence on the unit cell size is purely computational issue which is resolved by using primitive unit cell of finer size.

To better understand the reasons why accuracy of reverse PIM3 simulations depends on the unit cell length, a slightly mismatched lossless NTL has been modelled using a $1^{\circ}$ long unit cell. Its return loss at PIM3 frequency was the same as for a matched NTL simulated with $10^{\circ}$ long unit cell. Fig. 4 shows that mismatch of the NTL with $1^{\circ}$ long unit cell affects only PIM3 magnitude whereas the use of $10^{\circ}$ long unit cells alters the whole PIM3 length dependence in Figs. 3(b) and 4.

\section{VOLTAGE AND CURRENT DRIVEN NONLINEARITIES}

Several sources of nonlinearity of different nature may coexist in passive RF circuits and devices. Their individual or combined effect on PIM generation may vary depending on their physical origins and circuit configuration. The Harmonic Balance analysis has been adopted here to model PIM generation in NTL with combination of voltage and current driven nonlinearities. An equivalent circuit of constituent unit cell shown in Fig. 5 contains a lumped nonlinear capacitor and inductor. Capacitance $C$ and inductance $L$ are approximated by the quadratic polynomials: $C=C_{0}+C_{1} \cdot U_{C}+C_{2} \cdot U_{C}{ }^{2}$ and $L=L_{0}+L_{1} \cdot I_{L}+L_{2} \cdot I_{L}{ }^{2}$ where $U_{C}$ is voltage across the nonlinear capacitor and $I_{L}$ is current on the nonlinear inductor. 


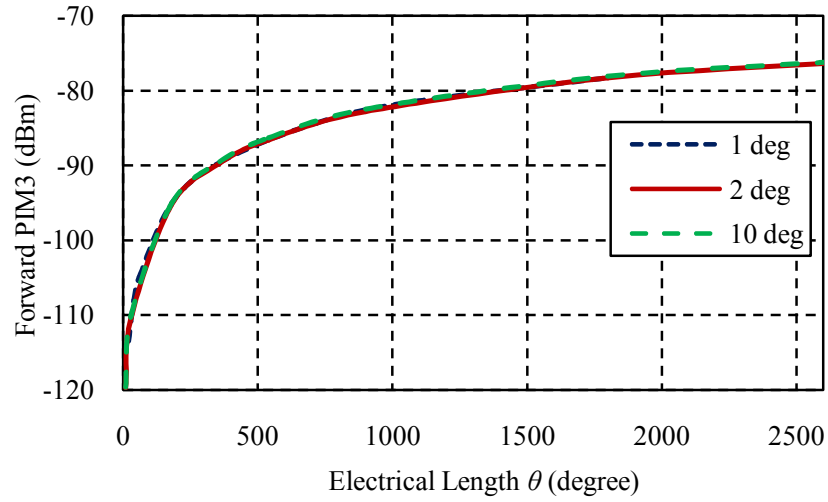

a)

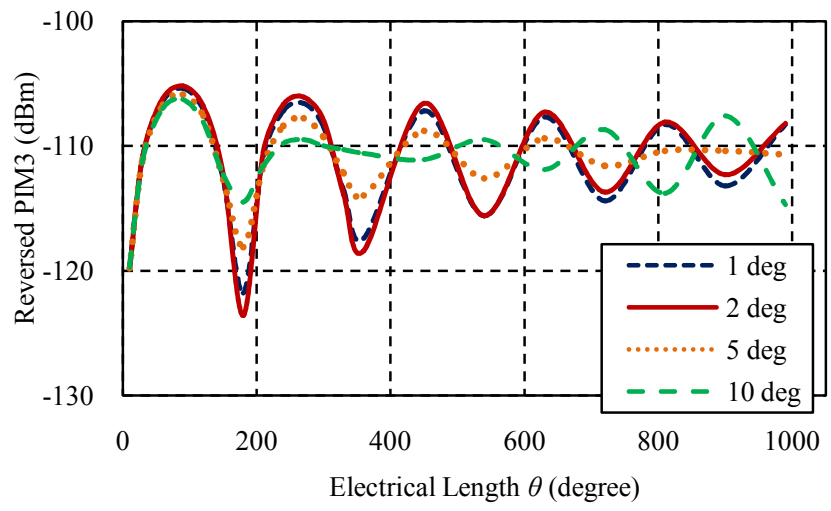

b)

Fig. 3. Forward (a) and reverse (b) PIM3 products versus electrical length of the NTL at several electrical lengths of the unit cell (shown in degrees).

Several scenarios have been examined, analysing NTL with each type of the nonlinearity separately and combined. Also, different models of the nonlinearities have been considered. The following circuit parameters were used to obtain commensurable levels of PIM3 products generated by different nonlinearities while keeping return loss low at both the carrier and PIM3 frequencies: $G=30 \mu \mathrm{S}$, $C_{0}=0.127 \mathrm{pF}, \quad C_{1}=2.1 \times 10^{-5} \mathrm{pF} / \mathrm{V}, \quad C_{2}=2.1 \times 10^{-9} \mathrm{pF} / \mathrm{V}^{2}$, $L_{0}=0.3 \mathrm{nH}, L_{1}=2.6 \mathrm{pH} / \mathrm{A}, L_{2}=1.3 \times 10^{-2} \mathrm{pH} / \mathrm{A}^{2}$.

Two-tone signals of frequencies $f_{1}=1.0 \mathrm{GHz}$ and $f_{2}=1.1$ $\mathrm{GHz}$ at power level of each tone $43 \mathrm{dBm}$ are injected at the NTL input. The results of PIM simulations are summarised in Table 1 for spectral components of frequencies $f_{2}-f_{l}=0.1 \mathrm{GHz}$ and $2 f_{1}-f_{2}=0.9 \mathrm{GHz}$ at the NTL input and output ports.

In the case of the second-order nonlinearity, even-order PIM products of frequencies $0.1 \mathrm{GHz}, 2.0 \mathrm{GHz}, 2.1 \mathrm{GHz}$ and $2.2 \mathrm{GHz}$ are dominant. However, there are also the third-order PIM products of frequencies $0.9 \mathrm{GHz}$ and $1.1 \mathrm{GHz}$ that arise as a result of the secondary processes of nonlinear mixing. As seen in Table 1, phase of the spectral components changes by $90^{\circ}$ at each step of the cascaded second-order mixing process.

It is noteworthy that PIM products generated by different types of nonlinearities exhibit different phases. This observation suggests a means for discriminating and identifying sources of nonlinearity by measuring phase of the PIM products. The fact that current and voltage driven

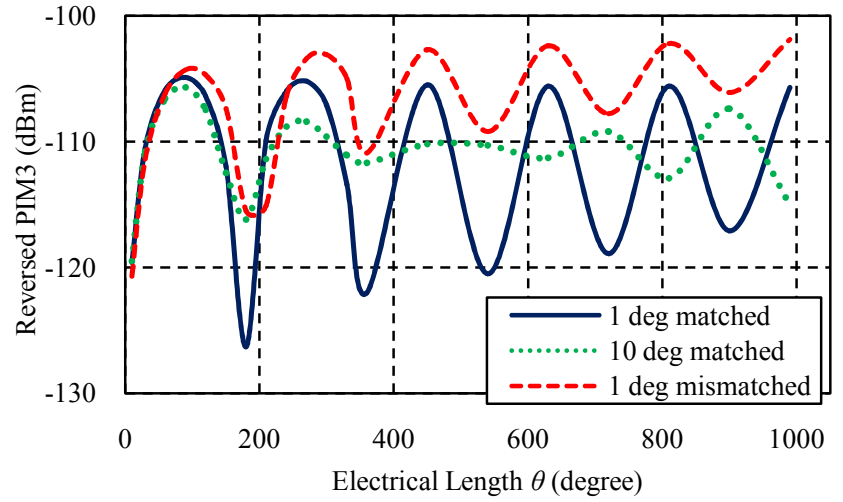

Fig. 4. Reverse PIM3 products versus electrical length of matched and mismatched NTL at different unit cell electrical lengths.

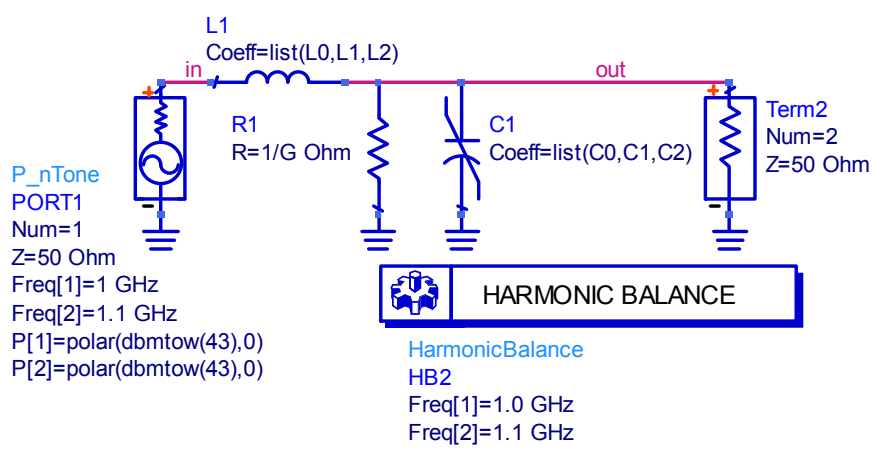

Fig. 5. ADS model of a constituent unit cell of NTL containing lumped nonlinear capacitor and inductor.

nonlinearities have differrent phase signatures may be also exploited for mitigation of reverse PIM by the destructive interference of the PIM products generated by nonlinearities of dissimilar physical nature. The detailed analysis of combined distributed nonlinearities in transmission lines based upon X-parameter approach will be reported elsewhere.

Table 1: Amplitude $(\mathrm{dBm})$ and phase (degrees) of spectral components at frequencies $f_{2}-f_{l}=0.1 \mathrm{GHz}$ and $2 f_{l}-f_{2}=0.9 \mathrm{GHz}$.

\begin{tabular}{|c|c|c|c|c|}
\hline \multirow{2}{*}{$\begin{array}{c}\text { Nonlinear } \\
\text { Model }\end{array}$} & \multicolumn{2}{|c|}{ Output PIM Products } & \multicolumn{2}{|c|}{ Input PIM Products } \\
\hline & $100 \mathrm{MHz}$ & $900 \mathrm{MHz}$ & $100 \mathrm{MHz}$ & $900 \mathrm{MHz}$ \\
\hline$C=C_{0}+C_{l} \cdot U_{C}$ & -59.6 & -124.0 & -59.6 & -124.0 \\
\hline$L=L_{0}$ & $\angle 89.8^{\circ}$ & $\angle 177.8^{\circ}$ & $\angle 89.6^{\circ}$ & $\angle 175.8^{\circ}$ \\
\hline$C=C_{0}$ & -59.7 & -124.2 & -59.7 & -124.2 \\
\hline$L=L_{0}+L_{l} \cdot I_{L}$ & $\angle-90.2^{\circ}$ & $\angle 178.2^{\circ}$ & $\angle 90^{\circ}$ & $\angle 0.2^{\circ}$ \\
\hline$C=C_{0}+C_{l} \cdot U_{C}$ & -100.2 & -118.1 & -53.6 & -118.2 \\
\hline$L=L_{0}+L_{l} \cdot I_{L}$ & $\angle 88.5^{\circ}$ & $\angle 173.0^{\circ}$ & $\angle 89.8^{\circ}$ & $\angle-4.7^{\circ}$ \\
\hline $\begin{array}{c}C=C_{0}+C_{2} \cdot U_{C}{ }^{2} \\
L=L_{0}\end{array}$ & 0 & $\begin{array}{c}-93.5 \\
\angle-92.1^{\circ}\end{array}$ & 0 & $\begin{array}{l}-93.5 \\
\angle-94^{\circ}\end{array}$ \\
\hline $\begin{array}{c}C=C_{0} \\
L=L_{0}+L_{2} \cdot I_{L}^{2}\end{array}$ & 0 & $\begin{array}{l}-93.6 \\
\angle-91.9^{\circ}\end{array}$ & 0 & $\begin{array}{l}-93.6 \\
\angle 90^{\circ}\end{array}$ \\
\hline $\begin{array}{c}C=C_{0}+C_{2} \cdot U_{C}^{2} \\
L=L_{0}+L_{2} \cdot I_{L}^{2}\end{array}$ & 0 & $\begin{array}{l}-87.6 \\
\angle-92^{\circ}\end{array}$ & 0 & $\begin{array}{l}-116.4 \\
\angle-178^{\circ}\end{array}$ \\
\hline
\end{tabular}




\section{CONCLUSION AND DISCUSSIONS}

A novel approach to the modelling of PIM generation in passive components with lumped and distributed nonlinearities is proposed. Based upon the formalism of Xparameters taking into account the effect of weak nonlinearities, it provides a unified framework for co-design of passive and active devices in RF front-end, antenna beamforming networks, phase shifters and others. The equivalent circuits of the canonical elements are readily incorporated in the nonlinear models, thus enabling evaluation of PIM impact on the performance of individual devices and their assemblies. An important advantage of the presented methodology is its compatibility with the industry-standard RF simulator Agilent ADS.

The major challenge in practical implementation of the proposed approach is concerned with experimental retrieval of $\mathrm{X}$-parameters of individual circuit elements. To our best knowledge commercial PIM testers and laboratory test instruments are inherently narrowband and do not allow for simultaneous vector measurements at the PIM and higher order harmonic frequencies. Alternatively, the existing nonlinear vector network analysers (NVNA) support broadband measurements of X-parameters with a range of stimuli but their dynamic range is insufficient for the PIM characterisation of practical circuits. Opportunities for adaptation of the X-parameters methodology to the PIM characterisation of passive devices using the existing test instruments will be further discussed in the presentation.

\section{ACKNOWLEDGEMENT}

This work has been carried out in the framework of Marie Curie European Industrial Doctorate (EID) programme ARTISAN jointly with Bell Labs Ireland. Dmitry Kozlov is supported by the Marie Curie EID Fellowship, grant No. 316426.

\section{REFERENCES}

[1] P. L. Lui, "Passive intermodulation interference in communication systems", Electron Electronics \& Communication Engineering Journal, Vol. 2, Issue 3, pp. 109-118, June 1990.

[2] J. Verspecht and D. Root, "Polyharmonic Distortion Modeling," IEEE Microwave Magazine, vol. 7, no. 3, pp. 44-57, June 2006.

[3] D. Gunyan, J Horn, Xu Jianjun, DE Root, "Nonlinear validation of arbitrary load X-parameter and measurement-based device models," IEEE MTT-S ARFTG Conference, Boston, MA, June 2009.

[4] M. Li, R. E. Amaya, R. G. Harrison and N. G. Tarr, "X-Parameter Measurement of Pulse-Compression Nonlinear Transmission Lines," Journal of Electrical and Computer Engineering, vol. 2010.

[5] S. Paul, K. Hüper, and J.A. Nossek, "Models for Nonlinear Transmission Lines," in Proc. ISCAS'93, Chicago IL, 3-6 May 1993, vol. 4, pp. 2287 - 2290

[6] D. E. Zelenchuk, A. P. Shitvov, A. G. Schuchinsky, and V. F. Fusco, "Passive intermodulation in finite lengths of printed microstrip lines," IEEE Transactions on Microwave Theory and Techniques, vol. 56, no. 11, Part 1, pp. 2426-2434, November 2008.

[7] A. P. Shitvov, D. E. Zelenchuk, A. G. Schuchinsky, and V. F. Fusco, "Passive intermodulation generation on printed lines: near-field probing and observations," IEEE Transactions on Microwave Theory and Techniques, vol. 56, no. 12, Part 2, pp. 3121-3128, December 2008. 